\title{
A Theoretical Estimate of the Pole-Equator Temperature Difference and a Possible Origin of the Near-Surface Shear Layer
}

\author{
Arnab Rai Choudhuri ${ }^{1}$
}

Received: 6 August 2020 / Accepted: 17 November 2020 / Published online: 15 February 2021

(C) The Author(s), under exclusive licence to Springer Nature B.V. part of Springer Nature 2021

\begin{abstract}
Convective motions in the deep layers of the solar convection zone are affected by rotation, making the convective heat transport latitude-dependent, but this is not the case in the top layers near the surface. We use the thermal wind balance condition in the deeper layers to estimate the pole-equator temperature difference. Surface observations of this temperature difference can be used for estimating the depth of the near-surface layer within which convection is not affected by rotation. If we require that the thermal wind balance holds in this layer also, then we have to conclude that this must be a layer of strong differential rotation and its characteristics which we derive are in broad agreement with the observational data of the near-surface shear layer.
\end{abstract}

Keywords Convection zone $\cdot$ Rotation $\cdot$ Velocity fields, interior $\cdot$ Helioseismology, observations

\section{Introduction}

Whether the surface temperature of the Sun has any variations with latitude is an interesting question both from theoretical and observational considerations. We point out that a simple order of magnitude estimate of the pole-equator temperature variation can be made from the thermal wind balance equation, which is the key equation in the theory of the meridional circulation. This equation is well known in the literature (see, for example, Kitchatinov, 2013; Karak et al., 2014, Section 5.2; Choudhuri, 2020) and a plot of this temperature variation calculated from the thermal wind balance equation is presented in a very recent paper (Matilsky, Hindman, and Toomre, 2020, Figure 13) without much detailed discussion of its significance. We show that an order of magnitude analysis of the thermal wind balance equation gives us a clue to understanding an enigmatic finding of helioseismology. Although the isorotation contours of solar differential rotation are nearly radial within the body of the convection zone, they bend towards the equator near the surface, giving rise to a near-surface

\footnotetext{
$凶$ A.R. Choudhuri

arnab@iisc.ac.in; arnabrch@gmail.com

1 Department of Physics, Indian Institute of Science, Bangalore, 560012, India
} 
Figure 1 A profile of the differential rotation inside the Sun obtained by helioseismology. We draw the reader's attention to the thick straight line parallel to the rotation axis starting from the point $\mathrm{O}$ at the bottom of the convection zone in the equatorial plane and extending to the point A just below the near-surface shear layer. This straight line will be used in Section 2 to estimate the centrifugal term. Adopted from Figure 26 of Basu (2016).

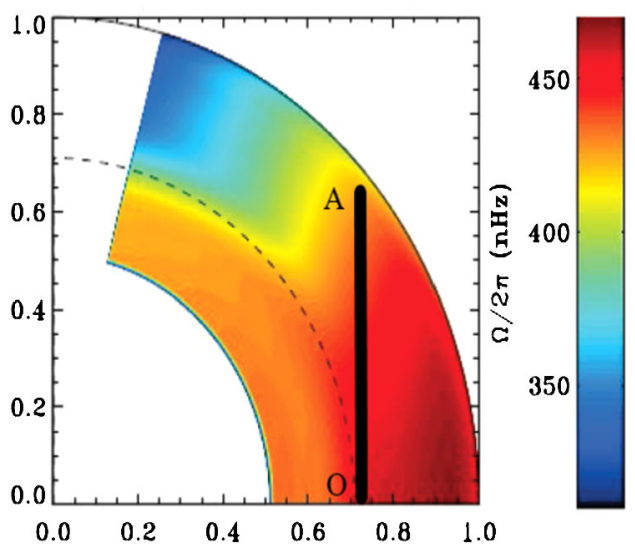

shear layer, which can be seen in Figure 1 of Howe (2009). This layer is clearly visible in the color plot of the differential rotation shown in Figure 1 (this paper).

The origin of this near-surface shear layer is still not properly understood. Well before this layer was mapped by helioseismology, the possible existence of such a layer was indicated from the observation that the rotation rate of the Sun inferred from the positions of sunspots differed by about 5\% with respect to the rate inferred from Doppler measurements and it was suggested that this was due to the effect of convection trying to make the specific angular momentum constant in the outer layers of the Sun (Foukal and Jokipii, 1975; Gilman and Foukal, 1979). There have been made some recent efforts to explain the near-surface shear layer on the basis of numerical simulation (Guerrero et al., 2013; Hotta, Rempel, and Yokoyama, 2015). Matilsky, Hindman, and Toomre (2019) suggested on the basis of their simulations that a strong gradient of density is essential for creating the near-surface shear layer. We propose a simple alternative explanation of this layer.

Heat is transported by convection in the outer layers of the Sun from about $0.7 \mathrm{R}_{\odot}$ to the solar surface. Convective motions are expected to be affected by the solar rotation if the convective turnover time $\tau_{\mathrm{c}}$ is more than or comparable to the solar rotation period, i.e. if

$$
\tau_{\mathrm{c}} \geq 25 \text { days. }
$$

Immediately below the solar surface, convection takes place in the form of granules with turnover times of the order of a few minutes. Since granular convection does not satisfy the condition given by Equation 1, we believe that convection below the surface till a depth, say $D$, will be completely unaffected by rotation. However, below this depth $D$, convection is likely to take place in the form of giant cells with much longer turnover times. Numerical simulations indeed show the existence of banana-shaped giant cells roughly aligned parallel to the rotation axis—-see Figure 1 in Brown et al. (2010) or Figure 3 in Gastine et al. (2014). Heat transport in such convection cells is clearly affected by rotation. Since the Coriolis force (which is crucial in producing the banana cells by acting on moving fluid parcels) is more effective in lower latitudes, we expect the effect of rotation to be more at the lower latitudes. Heat transport should be more efficient at higher latitudes, giving rise to a higher temperature at the poles. A hotter pole will tend to drive a meridional circulation equatorward at the solar surface (opposite to what is observed). This type of circulation driven by temperature differences on isochoric surfaces (i.e. surfaces of constant density) is often referred to as a thermal wind. It was realized nearly half a century ago that the effect of rotation 
on convection may make the heat transport in the Sun latitude-dependent and may produce a thermal wind (Durney and Roxburgh, 1971; Belvedere and Paterno, 1976).

The thermal wind is opposed by the centrifugal force term arising out of the differential rotation. With differential rotation mapped by helioseismology, one can now calculate this centrifugal force term fairly accurately. Within the main body of the convection zone, the centrifugal force term is expected to roughly balance the thermal wind term. In fact, the centrifugal force term must slightly overpower the thermal wind term to drive the meridional circulation in the correct direction. This balance is, however, thought to be upset in the layers just below the solar surface. It has been argued that the dissipative term, which is negligible inside the body of the convection zone compared to the centrifugal force term, becomes important in the layer near the surface and balances the centrifugal term, the thermal wind term being negligible there (Hotta, Rempel, and Yokoyama, 2015; Karak et al., 2014). We present a different point of view. We argue that the thermal wind term becomes even more important in the layer just below the surface and plays a crucial role in creating the nearsurface shear layer.

On the basis of a mean field model of the differential rotation and the meridional circulation, Kitchatinov and Ruediger (1995) concluded that the pole of the Sun has to be about $4 \mathrm{~K}$ hotter than the equator. An input from observations will be very crucial in deciding between the various alternative viewpoints. Ruediger $(1989$, p. 79$)$ has provided a summary of the early efforts made in determining the pole-equator temperature difference. Nearly all the authors reported an upper limit rather than an actual measurement. Such efforts have continued to be made (Kuhn, Libbrecht, and Dicke, 1988; Rast, Ortiz, and Meisner, 2008). In one of the last investigations we are aware of, Rast, Ortiz, and Meisner (2008) reported an excess temperature of $2.5 \mathrm{~K}$ in the polar region at the photospheric level. If this is true, then it provides strong support to our theoretical conjecture, as we shall point out. We hope that this important issue will be settled observationally in the near future.

An estimate of the pole-equator temperature difference from the thermal wind balance condition is presented in the next section (Section 2). Then Section 3 is devoted to determining the various characteristics of the near-surface shear layer by assuming that this balance condition holds in this layer also. Our conclusions are summarized in Section 4.

\section{An Order of Magnitude Estimate of the Pole-Equator Temperature Difference}

The well-known equation driving the meridional circulation (which is the equation of the azimuthal component of vorticity) can be derived from basic principles of fluid mechanics. See, for example, Choudhuri (2020) for a derivation of this equation. The two important source terms in this equation are the centrifugal term and the thermal wind term. The dissipation term within the convection zone turns out to be negligible compared to the centrifugal term estimated from helioseismology. This means that the centrifugal term cannot be balanced by the dissipation term in the interior of the convection zone and must be balanced by the thermal wind term in order to maintain a steady meridional circulation. This leads to the equation

$$
r \sin \theta \frac{\partial}{\partial z} \Omega^{2}=\frac{1}{r} \frac{g}{\gamma C_{V}} \frac{\partial S}{\partial \theta},
$$

where $S$ is the specific entropy (i.e. the entropy per unit mass), $z$ is measured parallel to the rotation axis starting from the equatorial plane and all the other symbols have their usual 
meanings. We may mention that Balbus et al. (2009) constructed a theoretical model of the differential rotation by solving this equation to find $\Omega(r, \theta)$ by assuming $S$ to be a function of $\Omega^{2}$. The validity of this assumption, however, is open to debate. We can use Equation 2 to make an order of magnitude estimate of the pole-equator temperature difference which we need for balancing the centrifugal term that would arise from the differential rotation measured by helioseismology.

We now try to estimate the magnitude of the centrifugal term, as given by the left hand side of Equation 2, below the shallow layer near the solar surface. For this purpose, we consider the straight line OA parallel to the rotation axis in Figure 1 . We note that $\Omega$ has the value $\Omega_{\mathrm{eq}} / 2 \pi \approx 460 \mathrm{nHz}$ at the point $\mathrm{O}$ of this line at the bottom of the convection zone and the value $\Omega_{\text {mid }} / 2 \pi \approx 420 \mathrm{nHz}$ at the point A where it reaches the bottom of the near-surface shear layer. This change in the value of $\Omega$ takes place over the length of the straight line OA, which is of order $\approx 0.75 \mathrm{R}_{\odot}$. Noting that the value of $r \sin \theta$ corresponding to any point on this straight line is very close to this, it is easy to argue that the left hand side of Equation 2 in the interior of the convection would approximately be equal to

$$
r \sin \theta \frac{\partial}{\partial z} \Omega^{2} \approx-\left[\Omega_{\mathrm{eq}}^{2}-\Omega_{\mathrm{mid}}^{2}\right]
$$

Substituting the values of $\Omega_{\text {eq }}$ and $\Omega_{\text {mid }}$, we get

$$
r \sin \theta \frac{\partial}{\partial z} \Omega^{2} \approx-\left[(460)^{2}-(420)^{2}\right] \times\left(2 \pi 10^{-9}\right)^{2} \mathrm{~s}^{-2} \approx-1.4 \times 10^{-12} \mathrm{~s}^{-2} .
$$

Next, we make an estimate of the right hand side of Equation 2. We note that the specific entropy of an ideal gas is given by

$$
S=C_{V} \ln T-(\gamma-1) C_{V} \ln \rho+K,
$$

where $K$ is a constant. The entropy difference between the equator and the pole on any isochoric surface (i.e. a surface of constant $\rho$ ) is

$$
\Delta S=C_{V} \ln \left(\frac{T_{\mathrm{eq}}}{T_{\text {pole }}}\right) .
$$

Taking $\Delta T$ to be the temperature excess of the pole with respect to the equator, we have

$$
\Delta S \approx-C_{V} \frac{\Delta T}{T_{\mathrm{S}}},
$$

where $T_{\mathrm{S}}$ is the temperature of our isochoric surface and we have made use of the approximation $\ln (1+x) \approx x$ for $|x| \ll 1$. We point out that our convention is that the direction of increasing $\theta$ is taken as the direction of increasing $S$ so that $\Delta S$ turns out to be negative. Since this entropy difference occurs over an angular separation $\pi / 2$, we have

$$
\frac{\partial S}{\partial \theta} \approx-2 C_{V} \frac{\Delta T}{\pi T_{\mathrm{S}}}
$$

It may be noted that $\partial S / \partial \theta$ implies differentiation at constant $r$, whereas the expression on the right hand is obtained over an isochoric surface. The justification for this is provided in Appendix A. Substituting Equation 6 in the right hand side of Equation 2, we get

$$
\frac{1}{r} \frac{g}{\gamma C_{V}} \frac{\partial S}{\partial \theta} \approx-\frac{2}{\pi \gamma} \frac{G \mathrm{M}_{\odot}}{\left(0.85 \mathrm{R}_{\odot}\right)^{3}} \frac{\Delta T}{T_{\mathrm{S}}},
$$


where we have taken $r$ to be given by $0.85 \mathrm{R}_{\odot}$ corresponding to the middle of the convection zone and have also used this to calculate $g$. If we now use the standard values of solar mass $\left(\approx 2 \times 10^{33} \mathrm{gm}\right)$ and radius $\left(\approx 7 \times 10^{10} \mathrm{~cm}\right)$, then we get (taking $\left.\gamma=5 / 3\right)$

$$
\frac{1}{r} \frac{g}{\gamma C_{V}} \frac{\partial S}{\partial \theta} \approx-2.4 \times 10^{-7} \frac{\Delta T}{T_{\mathrm{S}}} \mathrm{s}^{-2} .
$$

We note that Equation 4 gives the value of the left hand side of Equation 2 only inside the convection zone underneath the near-surface layer, whereas Equation 8 gives the value of the right hand side of Equation 2 for any isochoric surface to within an order of magnitude, provided $\Delta T / T$ is much smaller than 1. By equating Equation 4 and Equation 8, we arrive at

$$
\frac{\Delta T}{T_{\mathrm{S}}} \approx 5.8 \times 10^{-6} .
$$

If we take $T_{\mathrm{S}}$ equal to the temperature $5800 \mathrm{~K}$ at the photospheric surface, then we get a rather low value $\Delta T \approx 3.4 \times 10^{-2} \mathrm{~K}$. But should we use the photospheric temperature for $T_{\mathrm{S}}$ in Equation 9? As we point out, Equation 4 gives the magnitude of the centrifugal term underneath the near-surface shear layer. For the sake of consistency, we may expect Equation 8 would be equal to Equation 4 only if use $\Delta T / T$ for an isochoric surface below the near-surface shear layer. At what depth this isochoric surface should be is discussed in the next Section. We stress the rather non-intuitive fact that a centrifugal force resulting from a significant variation in $\Omega$ needs a very small pole-equator temperature difference to give rise to a thermal wind term to balance it.

\section{Physics of the Near-Surface Shear Layer}

As we move from a region inside the convection zone towards the solar surface, the convection cells would be of smaller size due to decrease in the pressure scale height and rotation would have less effect on the convection cells. While this is a gradual transition, we can make the following simplification. Below a certain depth $D$, we assume that Equation 1 is satisfied and convection is significantly affected by rotation. On the other hand, Equation 1 is not satisfied above $D$ and convection is unaffected by rotation. We now make an estimate of this depth $D$.

Within the convection zone, the temperature gradient $d T / d r$ is very nearly equal to the adiabatic gradient, the small difference between the two depending on the mixing length $l$ (Kippenhahn and Weigert, 1990, Section 7). Since the mixing length $l$ would be latitudedependent below $D$ due to the effect of rotation, the gradient $d T / d r$ would vary with latitude beneath $D$. On the other hand, the mixing length $l$ would be independent of latitude above $D$, leading us to conclude the $d T / d r$ would be the same at all latitudes in this layer near the surface. This means that the temperature would fall radially at the same rate at all latitudes in this layer. Suppose $\Delta T$ is the pole-equator temperature difference at the depth $D$. As we move from this depth $D$ towards the surface, the overall temperature would keep falling, but $\Delta T$ would still be the pole-equator temperature difference at the photospheric surface due to the temperature falling at the same rate at all latitudes. In other words, a pole-equator temperature difference at depth $D$ gets directly mapped to the photospheric surface, even though the overall temperature keeps falling. If $\Delta T$ is really 2.5 at the photospheric surface as claimed by Rast, Ortiz, and Meisner (2008), then we have to conclude that $\Delta T$ at the depth $D$ also should be $2.5 \mathrm{~K}$. 
Presumably, the depth $D$ would be in a region where the centrifugal term is given by Equation 4 and consequently Equation 9 holds. Taking $\Delta T \approx 2.5 \mathrm{~K}$, we conclude that the temperature of the isochoric surface at this depth would be of order

$$
T_{\mathrm{S}} \approx 4.3 \times 10^{5} \mathrm{~K}
$$

From the standard model of the convection zone (Spruit, 1974; Bahcall and Ulrich, 1988), we note that the temperature would have such a value at a radial distance of about $0.93 R_{\odot}$ from the solar center, which gives a depth of

$$
D \approx 4.9 \times 10^{4} \mathrm{~km}
$$

below the photospheric surface. Our contention is that, if the pole-equator temperature difference at the solar surface is really $2.5 \mathrm{~K}$, then the pole-equator temperature would continue to remain approximately $2.5 \mathrm{~K}$ till this depth $4.9 \times 10^{4} \mathrm{~km}$, in spite of the overall temperature changing by 2 orders of magnitude between the solar surface and this layer at depth $4.9 \times 10^{4} \mathrm{~km}$. If the temperature variation at the depth $D$ has to be imprinted to the surface, then $d T / d r$ has to be independent of latitude to a very high degree of precision. Can we expect this? We point out that the temperature difference even at the depth $D$ below which $d T / d r$ is latitude-dependent, as given by Equation 9, is extremely small. This suggests that the variation of $d T / d r$ with latitude has to be minuscule even in the deeper layers of the convection zone where convection is affected by rotation. For our ideas to work, the variation of $d T / d r$ with latitude has to be even much smaller than this in the top layer. Now that helioseismology is providing information about the amplitude of convective motions within the convection zone (Hanasoge, Duvall, and Sreenivasan, 2012; Greer et al., 2015), it will be important to address the question whether the mixing length theory with such convective amplitudes is consistent with such small values of $d T / d r$ variation with latitude caused by the solar rotation. It is beyond the scope of the present paper to analyze this extremely difficult problem. We merely point out these issues.

If $\Delta T$ remains the same but $T$ keeps falling as we move radially outward in this layer, certainly $\Delta T / T$ would keep increasing, making the thermal wind term given by Equation 8 stronger and stronger. To have an estimate of this term, we may take the temperature of an intermediate layer to calculate the thermal wind term. Taking $T_{\mathrm{S}} \approx 5 \times 10^{4} \mathrm{~K}$, the thermal wind term given by Equation 8 turns out to be

$$
\frac{1}{r} \frac{g}{\gamma C_{V}} \frac{\partial S}{\partial \theta} \approx-1.2 \times 10^{-11} \mathrm{~s}^{-2}
$$

The value of the thermal wind term in this top layer given by Equation 12 is clearly larger than the centrifugal term in the interior of the convection zone as given by Equation 4. If we want the centrifugal term and the thermal wind term to balance each other even in this thin layer, then clearly $d \Omega^{2} / d z$ has to be larger in this layer, showing the necessity of a nearsurface shear layer. We again refer to the thick solid line OA parallel to the rotation axis in Figure 1 . We have already pointed out that the value of $\Omega / 2 \pi$ is $\approx 420 \mathrm{nHz}$ at the point A below the near-surface layer we are considering. If $\Omega_{\text {top }} / 2 \pi$ is the value of the angular frequency at the point where this vertical straight line OA extended further would meet the solar surface, then the centrifugal term in this near-surface layer is easily seen to be of order

$$
r \sin \theta \frac{\partial}{\partial z} \Omega^{2} \approx-\frac{\mathrm{R}_{\odot}}{D}\left[(420)^{2}-\left(\frac{\Omega_{\mathrm{top}}}{2 \pi}\right)^{2}\right] \times\left(2 \pi 10^{-9}\right)^{2} \mathrm{~s}^{-2} .
$$


If we require that the centrifugal term and thermal wind term should be comparable even within this near-surface layer, then we have to equate Equation 12 and Equation 13. On using the value of $D$ given by Equation 11, this gives

$$
\frac{\Omega_{\mathrm{top}}}{2 \pi} \approx 390 \mathrm{nHz} \text {. }
$$

Looking at Figure 1, we note that the observational value is about $400 \mathrm{nHz}$. In other words, the jump in the value of $\Omega / 2 \pi$ according to our theoretical estimates is $\approx 30 \mathrm{nHz}$, whereas the observational jump is $\approx 20 \mathrm{nHz}$.

We thus find that our simple considerations give various important characteristics of the near-surface layer — such as its depth $D$ and the jump in $\Omega / 2 \pi$ within this layer-within a factor about 2 of the observational values. Since we use average values of various quantities in the top layer as in Equation 12 and Equation 13, one genuine concern is whether we are justified in doing this when quantities like the temperature vary by 2 orders of magnitude within this layer. We are right now carrying on a detailed quantitative analysis without such averaging and are looking at the question of how various things change on using other values of $D$ rather what is given in Equation 11. Results of this quantitative study will be presented in a paper currently under preparation.

\section{Conclusion}

It is generally believed that the centrifugal force term and thermal wind term approximately balance each other within the interior of the convection zone. From this balance condition, we estimate the pole-equator temperature difference. We argue that this temperature difference is appropriate for a layer at some depth below the surface and that this temperature difference remains the same as we move though the near-surface layer towards the surface, although the overall temperature keeps falling. Whether the thermal wind balance should hold within the near-surface layer as well is not a settled question. Hotta, Rempel, and Yokoyama (2015) argued that the centrifugal force term in this layer should be balanced by the turbulent dissipation term. We point out that the thermal wind term will become larger in this layer and propose the alternative viewpoint that this term will have to be balanced by the centrifugal force term. This suggests that the near-surface layer has to be a layer of strong differential rotation. The various characteristics of this layer which we infer agree with the properties of the near-surface shear layer measured by helioseismology.

We point out that we have not considered magnetic forces in our discussion. Although the magnetic forces are believed to drive the torsional oscillations (Chakraborty, Choudhuri, and Chatterjee, 2009) and the variations of the meridional circulation with the solar cycle (Hazra and Choudhuri, 2017), they presumably are not important in determining the mean characteristics of the large-scale flows.

We have argued that the explanation of the near-surface shear layer involves heat transfer and fluid dynamics in the top layers of the solar convection zone. This layer is responsible for the difference between the rotation speeds of sunspots and the gas at the solar surface. Whether this layer also plays a role in the dynamo process for generating solar magnetic fields remains unclear. We know that the differential rotation of the Sun is responsible for the generation of the toroidal field of the Sun (Choudhuri, 2011; Karak et al., 2014). Since the strong superadiabatic temperature gradient in the top layers of the convection zone would make magnetic buoyancy particularly effective there (Moreno-Insertis, 1983), it is generally believed that any magnetic fields produced in the top layers would rise up quickly without 
leaving much time for field amplification. Numerical simulations based on the assumption that the toroidal field is generated in the tachocline and remains partially anchored there match observational data such as Joy's law quite well (D'Silva and Choudhuri, 1993).

The value of the pole-equator temperature difference at the surface is crucial in estimating the characteristics of the near-surface shear layer. On using the value $2.5 \mathrm{~K}$, as reported by Rast, Ortiz, and Meisner (2008), we find that the characteristics of this layer which we obtain are in reasonable agreement with measurements from helioseismology. In case there is no pole-equator temperature difference at the surface, then our idea will clearly not work. Due to the difficulties in treating the near-surface layer realistically in numerical simulations, it is not possible to draw any firm conclusion about this temperature difference from simulations (Bidya Karak, private communication). We hope that the temperature difference at the solar surface will be firmly established by independent measurements of different groups in the near future.

Acknowledgements I thank Dipankar Banerjee, Gopal Hazra, Bibhuti Kumar Jha, Leonid Kitchatinov and Bidya Karak for valuable discussions. My research was supported in part by a J.C. Bose Fellowship awarded by the Department of Science and Technology, Government of India. I thank an anonymous referee for very valuable comments, which helped greatly in improving the paper.

Disclosure of Potential Conflicts of Interest The author declares that he has no conflicts of interest.

Publisher's Note Springer Nature remains neutral with regard to jurisdictional claims in published maps and institutional affiliations.

\section{Appendix A: On Differentiating Entropy Along an Ioschore}

The difference of specific entropy $\Delta S$ given by Equation 5 refers to an isochore over which $\rho$ does not vary. If $l$ is the length measured along an isochore, then the entropy difference between two points on an isochore should be given be

$$
\Delta S=\frac{d S}{d l} \Delta l
$$

From the chain rule of partial differentiation, it follows that

$$
\frac{d S}{d l}=\left(\frac{\partial S}{\partial r}\right)_{\theta} \frac{d r}{d l}+\left(\frac{\partial S}{\partial \theta}\right)_{r} \frac{d \theta}{d l} .
$$

It is easy to argue that the first term in this equation is going to be negligible. Efficient convection tends to homogenize $S$ in the radial direction so that $(\partial S / \partial r)_{\theta} \approx 0$. Since the rotational flattening of the Sun is very small, we expect the isochoric surfaces to be very nearly spherical so that $d r / d l$ is also expected to be very small. Since the first term is a product of two small terms, we can write

$$
\frac{d S}{d l} \approx\left(\frac{\partial S}{\partial \theta}\right)_{r} \frac{d \theta}{d l} .
$$

Substituting this in Equation A.1, we get

$$
\Delta S \approx\left(\frac{\partial S}{\partial \theta}\right)_{r} \Delta \theta .
$$

If we take $\Delta \theta=\pi / 2$ for the pole-equator difference, then Equation A.2 leads to Equation 6. 


\section{References}

Bahcall, J.N., Ulrich, R.K.: 1988, Solar models, neutrino experiments, and helioseismology. Rev. Mod. Phys. 60, 297. DOI. ADS.

Balbus, S.A., Bonart, J., Latter, H.N., Weiss, N.O.: 2009, Differential rotation and convection in the Sun. Mon. Not. Roy. Astron. Soc. 400, 176. DOI. ADS.

Basu, S.: 2016, Global seismology of the Sun. Living Rev. Solar Phys. 13, 2. DOI. ADS.

Belvedere, G., Paterno, L.: 1976, Large scale circulation in the convection zone and solar differential rotation. Solar Phys. 47, 525. DOI. ADS.

Brown, B.P., Browning, M.K., Brun, A.S., Miesch, M.S., Toomre, J.: 2010, Persistent magnetic wreaths in a rapidly rotating Sun. Astrophys. J. 711, 424. DOI. ADS.

Chakraborty, S., Choudhuri, A.R., Chatterjee, P.: 2009, Why does the Sun's torsional oscillation begin before the sunspot cycle? Phys. Rev. Lett. 102, 041102. DOI. ADS.

Choudhuri, A.R.: 2011, The origin of the solar magnetic cycle. Pramana 77, 77. DOI. ADS.

Choudhuri, A.R.: 2020, The meridional circulation of the Sun: Observations, theory and connections with the solar dynamo. DOI. arXiv. ADS.

D'Silva, S., Choudhuri, A.R.: 1993, A theoretical model for tilts of bipolar magnetic regions. Astron. Astrophys. 272, 621. ADS.

Durney, B.R., Roxburgh, I.W.: 1971, Inhomogeneous convection and the equatorial acceleration of the Sun. Solar Phys. 16, 3. DOI. ADS.

Foukal, P., Jokipii, J.R.: 1975, On the rotation of gas and magnetic fields at the solar photosphere. Astrophys. J. Lett. 199, L71. DOI. ADS.

Gastine, T., Yadav, R.K., Morin, J., Reiners, A., Wicht, J.: 2014, From solar-like to antisolar differential rotation in cool stars. Mon. Not. Roy. Astron. Soc. 438, L76. DOI. ADS.

Gilman, P.A., Foukal, P.V.: 1979, Angular velocity gradients in the solar convection zone. Astrophys. J. 229, 1179. DOI. ADS.

Greer, B.J., Hindman, B.W., Featherstone, N.A., Toomre, J.: 2015, Helioseismic imaging of fast convective flows throughout the near-surface shear layer. Astrophys. J. Lett. 803, L17. DOI. ADS.

Guerrero, G., Smolarkiewicz, P.K., Kosovichev, A.G., Mansour, N.N.: 2013, Differential rotation in solar-like stars from global simulations. Astrophys. J. 779, 176. DOI. ADS.

Hanasoge, S.M., Duvall, T.L., Sreenivasan, K.R.: 2012, Anomalously weak solar convection. Proc. Natl. Acad. Sci. 109, 11928. DOI. ADS.

Hazra, G., Choudhuri, A.R.: 2017, A theoretical model of the variation of the meridional circulation with the solar cycle. Mon. Not. Roy. Astron. Soc. 472, 2728. DOI. ADS.

Hotta, H., Rempel, M., Yokoyama, T.: 2015, High-resolution calculation of the solar global convection with the reduced speed of sound technique. II. Near surface shear layer with the rotation. Astrophys. J. 798, 51. DOI. ADS.

Howe, R.: 2009, Solar interior rotation and its variation. Living Rev. Solar Phys. 6, 1. DOI. ADS.

Karak, B.B., Jiang, J., Miesch, M.S., Charbonneau, P., Choudhuri, A.R.: 2014, Flux transport dynamos: from kinematics to dynamics. Space Sci. Rev. 186, 561. DOI. ADS.

Kippenhahn, R., Weigert, A.: 1990, Stellar Structure and Evolution. ADS.

Kitchatinov, L.L.: 2013, Theory of differential rotation and meridional circulation. In: Kosovichev, A.G., de Gouveia Dal Pino, E., Yan, Y. (eds.) Solar and Astrophysical Dynamos and Magnetic Activity, IAU Symposium 294, 399. DOI. ADS.

Kitchatinov, L.L., Ruediger, G.: 1995, Differential rotation in solar-type stars: revisiting the Taylor-number puzzle. Astron. Astrophys. 299, 446. ADS.

Kuhn, J.R., Libbrecht, K.G., Dicke, R.H.: 1988, The surface temperature of the Sun and changes in the solar constant. Science 242, 908. DOI. ADS.

Matilsky, L.I., Hindman, B.W., Toomre, J.: 2019, The role of downflows in establishing solar near-surface shear. Astrophys. J. 871, 217. DOI. ADS.

Matilsky, L.I., Hindman, B.W., Toomre, J.: 2020, Revisiting the Sun's strong differential rotation along radial lines. Astrophys. J. 898, 111. DOI. ADS.

Moreno-Insertis, F.: 1983, Rise times of horizontal magnetic flux tubes in the convection zone of the Sun. Astron. Astrophys. 122, 241. ADS.

Rast, M.P., Ortiz, A., Meisner, R.W.: 2008, Latitudinal variation of the solar photospheric intensity. Astrophys. $J .673,1209$. DOI. ADS.

Ruediger, G.: 1989, Differential Rotation and Stellar Convection. Sun and the Solar Stars. ADS.

Spruit, H.C.: 1974, A model of the solar convection zone. Solar Phys. 34, 277. DOI. ADS. 